\title{
Afectación del aprendizaje en los alumnos ante el Covid-19
}

\section{Sergio Gabriel Ordoñez Sánchez}

sergio.ordonez@correo.buap.mx

Benemérita Universidad Autónoma de Puebla

Gerardo Hernández Barrena

gerardo.hernandez@correo.buap.mx

Benemérita Universidad Autónoma de Puebla

Axel Jesús Escoto Flores

axel.escoto@alumno.buap.mx

Benemérita Universidad Autónoma de Puebla

Elizabeth Cabrera Tenorio

elizabeth.cabrera@utpuebla.edu.mx

Universidad Tecnológica de Puebla

\section{RESUMEN}

En la actualidad nos encontramos en un momento de "crisis" en el mundo, en todos los aspectos de la vida como lo es la educación, la familia, la economía, el bienestar y la salud. Cualquiera de estas variantes se ha visto afectada por la actual enfermedad Covid19 de una manera en la que se ha tenido que cambiar la dinámica de las cosas completamente, a los estudiantes les tocó vivir un cambio en la manera de aprendizaje ya que todo ha sido realizado en una modalidad en línea. La presente investigación tiene por objetivo, observar como el alumno ha sido afectado en su aprendizaje y determinar la vulnerabilidad y el sentir emocional del estudiante referente a como se desarrolla en la actualidad su vida académica derivado de la pandemia, la metodología utilizada es de tipo cuantitativo y cualitativo, deductivo, no experimental; aplicando un instrumento tipo encuesta y obteniendo como principales resultados que el encierro y la falta de socialización puede traer serias consecuencias en la salud mental de los estudiantes universitarios.

Palabras clave: Educación; Alumno; Emoción; Aprendizaje 


\title{
Affection of learning in students consequence of Covid-19
}

\begin{abstract}
We are currently in a time of "crisis" in the world, in all aspects of life such as education, family, economy, well-being and health. Any of these variants has been affected by the current disease Covid-19 in a way that has had to change the dynamics of things completely, the students had to experience a change in the way of learning since everything has been done in an online mode. The objective of this research is to observe how the student has been affected in their learning and determine the vulnerability and emotional feeling of the student regarding how their academic life derived from the pandemic is currently developing, the methodology used is quantitative and qualitative, deductive, not experimental; applying a survey type instrument and obtaining as main results that confinement and lack of socialization can have serious consequences on the mental health of university students.
\end{abstract}

Keywords: Education; Student; Emotion; Learning

Artículo recibido: 03 nov. 2020

Aceptado para publicación: 07 dic. 2020

Correspondencia sergio.ordonez@correo.buap.mx

Conflictos de Interés: Ninguna que declarar 


\section{INTRODUCCIÓN}

En diciembre de 2019 se identificaron casos de neumonía que fueron originados por un nuevo tipo de coronavirus esto en Wuhan (China), a este tipo se le dieron distintas denominaciones: 2019-nCoV según la OMS y SARS-CoV-2 según el Comité Internacional de Taxonomía de Virus. El 7 de enero de 2020, las autoridades chinas reconocieron este virus como el causante de dichos casos de neumonía (Juárez, 2020).

El coronavirus es parte de una familia de virus y estos causan enfermedades las cuales pueden ser tanto en humanos como en animales, en los humanos es muy común que este virus ocasione enfermedades, molestias e infecciones respiratorias sin embargo también puede derivar en enfermedades más graves, como lo es el síndrome respiratorio de oriente medio (MERS) y el síndrome respiratorio agudo severo (SRAS). En específico este nuevo tipo de coronavirus que se ha descubierto, causa una enfermedad denominada Covid-19 (OMS, 2020), ya que Co abrevia la palabra "corona", vi hace referencia a la palabra "virus", la letra $d$ que significa enfermedad en inglés "disease" y 19 nos dice el año en el que surgió.

Hasta el 7 de diciembre de 2020 varios casos han sido registrados en todo el mundo, según la Organización Mundial para la Salud (OMS): se han reportado 65, 900,000 casos a nivel global y de esos han muerto 1,520,000 (2.30\%), lo que la convierte en una enfermedad catalogada como emergencia de salud pública a nivel mundial (EFE, 2020 ).

Existen diversos síntomas de esta enfermedad, estos se manifiestan de manera leve y con el tiempo, comienzan gradualmente a complicarse.

En algunos casos las personas no presentan síntomas o son asintomáticas. Los síntomas principales son la fiebre, tos seca, cansancio, sin embargo, existen síntomas menos frecuentes que también pueden ser indicadores de la enfermedad, los cuales son: dolor de garganta, diarrea, perdida de gusto o del olfato, erupciones cutáneas y cambios de color en los dedos de las manos y los pies, entre otras (OMS, 2020).

Sin embargo la mortalidad de esta enfermedad es muy baja ya que una de cada cinco personas acaban realmente presentando síntomas graves, las cuales derivan en dificultades para respirar, y si esas personas padecen alguna enfermedad previa como hipertensión arterial, problemas cardiacos o pulmonares, diabetes o cáncer, puede existir una gran probabilidad de presentar síntomas más severos; sin embargo los números 
arrojan que alrededor del $80 \%$ de los infectados se recuperan de manera exitosa, sin necesidad si quiera de acudir a un hospital. (OMS, 2020)

El problema de esta enfermedad Covid-19 no radica en la mortalidad, si no en la propagación de la misma, ya que la propagación de esta enfermedad es viral, lo cual significa que una persona puede contraer Covid-19 por contacto con otra que esté infectada por dicho virus, la propagación de persona a persona es a través de una micro sustancia denominada gotícula que usualmente sale por la nariz o la boca al momento de hablar, estornudar o toser; es decir, si la persona que no está infectada inhala las gotículas procedentes de una persona que si está infectada contraerá la enfermedad, así mismo estas gotículas caen sobre objetos y superficies como sillas, computadoras, controles, almohadas, de tal modo que una persona no infectada llega a tocar dichas superficies, también puede contraer la enfermedad. (OMS, 2020).

Este método de propagación de la enfermedad ha llevado al mundo entero a declararse en un modo de alerta, porque si bien, la mortalidad de la enfermedad es muy baja, ya que de los 7.594 miles de millones de personas que somos en el mundo, si la enfermedad se propagara en todas las personas, quiere decir que por lo menos terminaría con la vida de 151 millones de personas en el planeta.

Se llama pandemia a la propagación mundial de una nueva enfermedad, esto sucede cuando surge un nuevo virus que se propaga por el mundo y la mayoría de las personas no tienen inmunidad contra él, llevándonos a un aislamiento mundial (OMS, 2020).

El aislamiento es la medida que todos los gobiernos y países del mundo, han adoptado para intentar controlar la propagación de la enfermedad; esta medida es con el fin de evitar la infección a otras personas y consiste en el aislamiento social y laboral, es decir, no salir de casa para ir al trabajo, a la escuela o a los lugares públicos. Con anterioridad ya habían existido pandemias y aislamientos sin embargo, nunca nos había tocado vivir una que afectara a todo el mundo, principalmente en la economía, en el empleo, en el desarrollo y uno de los factores más importantes en el de educación, todo esto porque los estudiantes así como todas las personas han dejado de ir a los lugares donde realizan sus actividades del día a día, para cambiar a una modalidad denominada a distancia, la cual consiste en realizar todas las actividades, congresos, trabajos, estudios a través de una computadora, un monitor, la televisión, todo por medio de la red de internet. Esto ha cambiado la realidad en la que se vivía, lo cual ha afectado a los estudiantes de manera considerable 
porque se ha pasado de estudiar en las instalaciones escolares a estudiar desde casa y esto, por ende, ha generado un sinfín de sentimientos y emociones en los estudiantes universitarios. Es por ello que es de vital importancia, darnos cuenta como se sienten y como han sido afectados, esto para poder desarrollar algún plan de reincorporación y de bienestar emocional.

Según la Secretaria de Salud, en este tipo de suceso epidemiológico, las personas tienen un aumento de angustia, miedo, pánico o terror, así como ansiedad e incertidumbre de su futuro en el caso de las epidemias, el riesgo de problemas de salud mental (estado de bienestar) crece debido a las medidas de aislamiento social, restricción de movilidad y reducción del contacto físico. (Asociación Mexicana de Industrias de Investigación Farmacéutica, A.C., 2020)

La actual pandemia y aislamiento ha derivado efectos devastadores en la educación y el desarrollo de los estudiantes de todos los niveles en el mundo, como principales factores se encuentra la pérdida de clases presenciales y una difícil adaptación a un modelo de estudio a distancia.

Estas afectaciones se ven incrementadas con otros factores como, por ejemplo: falta de internet, falta de un equipo de cómputo, falta de cable, disponibilidad de tiempos y en su caso algunas escuelas han tenido que cerrar sus puertas derivado de la afectación económica que la pandemia trajo consigo (ONU México, 2020).

La mayor parte de los planteles en México, no están preparados para dar una buena educación a distancia, un número considerable de los docentes no están capacitados para enseñar de manera remota, sin contar los factores de problemas de infraestructura, de capacitación de los docentes, de la adaptación de los materiales de enseñanza satisfactoria a través de la forma remota; con todas estas variantes. Se puede observar lamentablemente que el sistema educativo no está preparado para enfrentar una contingencia, que implica el cierre total de las escuelas en nuestro país y por ende se afecta a los jóvenes en mayor medida, al no recibir una educación de calidad (Ruiz, 2020 ).

Al investigar cómo está afectando la pandemia a los jóvenes en nuestro país, un estudio del Centro de Opinión Pública (COP) de la Universidad del Valle de México, revela que el $44 \%$ han visto perjudicados en su estado emocional a causa del aislamiento por COVID-19 (Prensa UVM, 2020), y no solo eso, ya que la Secretaría de Educación Superior (SEP) consideró que aproximadamente el $8 \%$ de estudiantes de nivel superior, 
abandonaron sus estudios a causa de la pandemia de coronavirus (Redacción Animal Político, 2020).

Con estos datos se pretende analizar qué es lo que está pasando en los estudiantes, en su desarrollo académico y su bienestar emocional; además de conocer cómo es que la pandemia los hace sentir. Sin duda alguna es de vital importancia escuchar a los jóvenes, para dar una respuesta más inclusiva a la enfermedad que actualmente atravesamos.

"La pandemia tiene una repercusión muy adversa en los jóvenes. No sólo merma su empleo y futuro profesional, sino que menoscaba en gran medida su educación y formación, y, por ende, su bienestar mental. No podemos permitir que eso suceda" Guy Ryder, Director General de la OIT.

\section{ESTRATEGIAS METODOLÓGICAS O MATERIALES Y MÉTODOS}

El objetivo general de esta investigación, es conocer cómo se sienten los alumnos de la Facultad de Contaduría Pública de la Benemérita Universidad Autónoma de Puebla después de haber concluido el periodo agosto - diciembre de 2020 en sus clases a distancia, producto de la pandemia, para determinar la efectividad del proceso de aprendizaje.

La investigación se realizó bajo un método mixto (deductivo-inductivo), analizando y explicando los conceptos de los temas, de lo general a lo particular.

Se trata de una investigación exploratoria, debido a que se llevó a cabo con un enfoque cualitativo y cuantitativo, haciendo un análisis de fuentes secundarias como son: libros, periódicos, revistas, y páginas electrónicas.

Los métodos de estudio se llevaron a cabo por medio de un instrumento tipo encuesta.

Para el estudio se consideró una muestra de 500 alumnos de un grupo de 3,500 estudiantes de la Facultad de Contaduría Pública de la Benemérita Universidad Autónoma de Puebla, durante el periodo agosto - diciembre de 2020.

Se elaboraron graficas tipo pastel, para mostrar los resultados en forma porcentual y se procede a la eliminación de los extremos de la muestra con valores máximos y mínimos para reducir el grado de error y sesgo.

\section{RESULTADOS Y DISCUSIÓN}

La encuesta fue realizada con el fin de conocer la situación de los estudiantes de la Facultad de Contaduría Pública de la Benemerita Universidad Autonoma de Puebla, estos resultados se muestran en las siguientes gráficas para su mejor entendimiento. 
De las encuestas realizadas el $72.7 \%$ de los estudiantes son del genero femenino y solo el $27.3 \%$ del genero masculino y asi mismo, las edades de los encuestados oscilan entre los 19 y 26 años.

\section{1. ¿Qué opinas en general sobre la educación a distancia?}

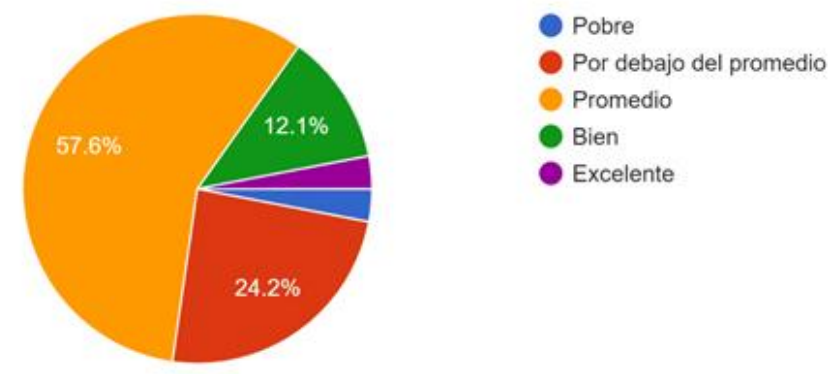

Figura 1. Fuente: Elaboración propia (2020).

Los resultados de la pregunta número 1 indican que para la mayoría de los estudiantes la educación a distancia no ha sido tan buena pero tampoco tan mala, el 57.6\% de los estudiantes dijo que la educación ha sido promedio, para el 24.2\% la educación ha sido mala, y para el $12.1 \%$ ha sido buena. (Figura 1).

4. ¿Cuánto tiempo dedicas cada dia en promedio a la educación a distancia?

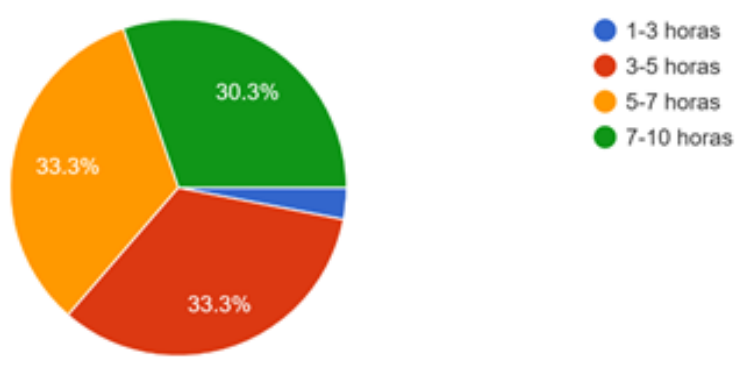

Figura 2. Fuente: Elaboración propia (2020).

El 33.3\% de los estudiantes dicen estudiar de 5 a 7 horas al día, el mismo porcentaje lo tiene la opción de 3 a 5 horas, alrededor de 22 estudiantes estudian de 3 a 7 horas diarias, el 3\% dijo estudiar solo de 1 a 3 horas al día, miestras que el 30.3\% de 7 a 10 horas al día (Figura 2). 


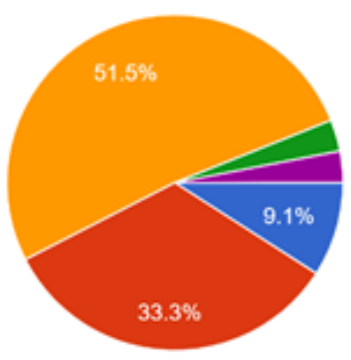

No ha sido efectivo en absoluto

Ligeramente efectivo

Moderadamente eficaz

Muy eficaz

- Extremadamente eficaz

Figura 3. Fuente: Elaboración propia (2020).

Para la mayoría de los estudiantes de nuestra encuesta con el $51.5 \%$ dicen que el aprendizaje a distancia es moderadamente eficaz, el 33.3\% de los estudiantes dice que es ligeramente efectivo para ellos, la opción no ha sido efectivo en absoluto tiene el $9.1 \%$, y solo el 3\% contesto que el aprendizaje es muy eficaz, la misma cifra aplica para la opción extremadamente eficaz (Figura 3).

7. ¿Qué tan estresante es para ti la educación a distancia durante la pandemia de COVID-19?

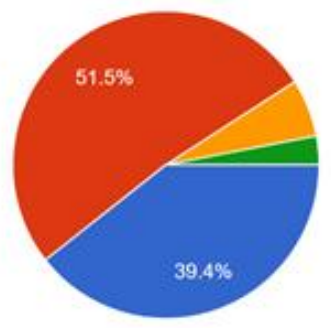

Figura 4. Fuente: Elaboración propia (2020).

Para el $51.5 \%$ de los estudiantes es medio estresante la educación a distancia, para el $39.4 \%$ dice que es muy estresante para ellos, el $6.1 \%$ de los estudiantes dice que solo es un poco estresante y el 3\% se muestra indiferente ante la situación (Figura 4).

11. ¿Qué tan preocupado está por su salud fisica y emocional?
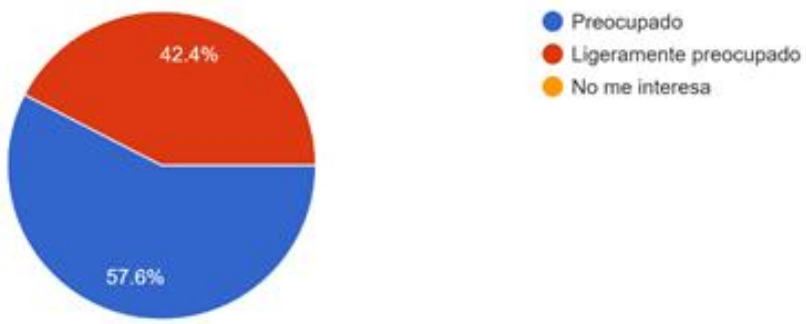

No me interesa

Figura 5. Fuente: Elaboración propia (2020). 
La salud física y emocional de los estudiantes es muy importante para ellos, ya que el $57.6 \%$ está muy preocupado por su salud. Tan solo el $42.4 \%$ esta ligeramente preocupado (Figura 5). 18. ¿Qué tan ansioso se encuentra acerca de su progreso en la licenciatura debido al
distanciamiento fisico y social?

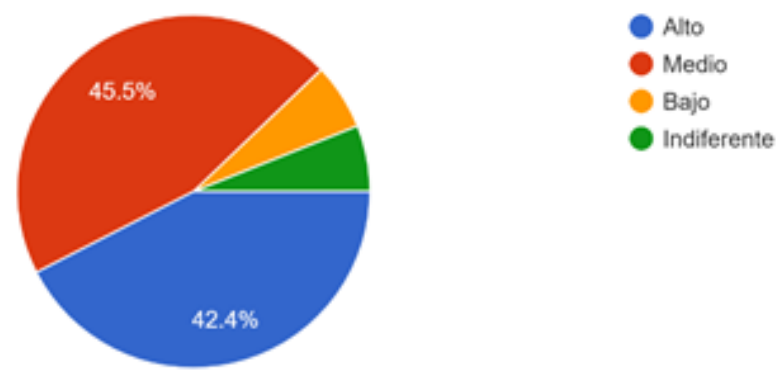

Figura 6. Fuente: Elaboración propia (2020).

Como vemos hay un $45.5 \%$ de grado de ansiedad, de acuerdo al progreso que se tiene en la licenciatura por parte de los estudiantes (Figura 6).

\section{0. ¿Cómo sientes tu entorno familiar en tu hora clase?}

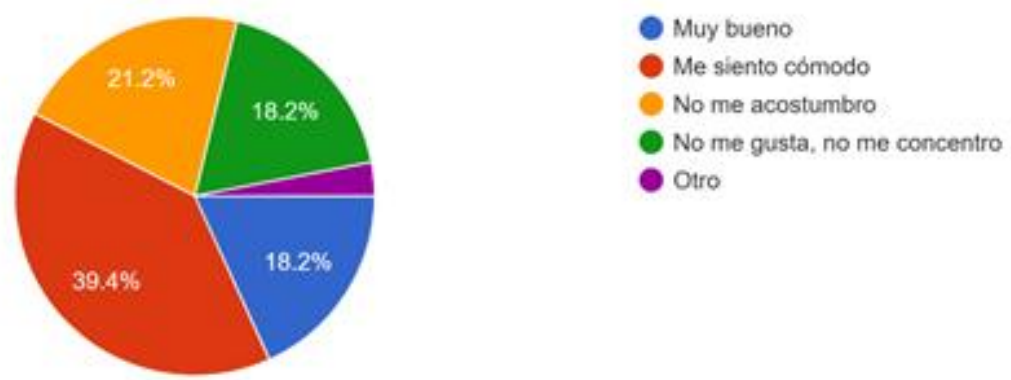

Figura 7. Fuente: Elaboración propia (2020).

Esta grafica nos indica que el $39.4 \%$ de estudiantes no se siente cómodo en su entorno familiar, y con un $21.2 \%$ contestaron que no se acostumbran. El 18.2\% de los estudiantes no se concentran, lo que conlleva a una falta de aprovechamiento en sus horas de clases. (Figura 7).

\section{CONCLUSIÓN O CONSIDERACIONES FINALES}

Como conclusión podemos afirmar que, en base a los resultados de la encuesta aplicada, los alumnos de la Facultad de Contaduría Pública, muestran ansiedad y preocupación por su salud. 
Conservar la salud mental de los estudiantes es uno de los principales retos que deben afrontar las Instituciones de Educación Superior (IES) en el contexto actual, si lo que se busca es hacer que esta situación sea más idónea y no tenga impactos negativos, como el abandono y la deserción en perjuicio de la eficiencia terminal.

Es importante que las IES desarrollen instrumentos para evaluar la salud mental y emocional de sus estudiantes, profesores y personal administrativo, con el fin de identificar aquellos miembros de la comunidad universitaria, afectados por el periodo de confinamiento obligatorio y el estrés asociado a la crisis que trajo el Covid-19. Así mismo, diseñar una estrategia que brinde acompañamiento y atención a aquellos que más lo requieran.

Se considera que la situación que se vive actualmente es complicada en todos los aspectos, y sin duda alguna, repercute en el ámbito educativo, ya que resulta complicado un aprendizaje en línea, debido a que no se cuenta con el contacto directo docente-alumno, interacción que resulta muy importante en el proceso de enseñanza-aprendizaje acostumbrado.

Finalmente, lo que se debe tener claro es que, en el aspecto educativo, nos debemos preparar para dar un paso adelante con la modalidad a distancia, las nuevas tecnologías, el aprendizaje autodidacta, el diseño de planes y programas de estudio integrales con herramientas de salud mental, fortalecer las capacidades de los y las docentes, para brindar una educación de calidad y accesible para todos los estudiantes, de cualquier nivel e institución.

\section{REFERENCIA BIBLIOGRAFICA}

Asociación Mexicana de Industrias de Investigación Farmacéutica, A.C. (1 de agosto de 2020). La salud mental frente a la COVID-19. Recuperado el 8 de diciembre de 2020, de https://amiif.org/la-salud-mental-frente-al-covid-19/EFE. (06 de Diciembre de 2020 ). OMS reporta 65.9 millones casos de covid-19 en el mundo. Obtenido de https://www.milenio.com/internacional/oms-coronavirus-mundo-roza-66-millonescontagios

Juárez, M. L. (20 de Marzo de 2020). Covid-19, una emergencia de salud pública mundial. Obtenido de https://www.ncbi.nlm.nih.gov/pmc/articles/PMC7102523/

OMS. (2020). Preguntas y respuestas sobre la enfermedad por coronavirus (COVID19). Obtenido de https://www.who.int/es/emergencies/diseases/novel- 
coronavirus-2019/advice-for-public/q-a-

coronaviruses\#: :text=La\%20COVID\%E2\%80\%9119\%20es\%20la,China)\%20e n\%20diciembre\%20de\%202019.

ONU México. (11 de Agosto de 2020). La COVID-19 interrumpe la educación de más del 70\% de los jóvenes. Obtenido de https://coronavirus.onu.org.mx/la-covid-19interrumpe-la-educacion-de-mas-del-70-de-los-jovenes

Prensa UVM. (20 de Julio de 2020). COVID-19 afecta estado emocional y económico de jóvenes en México: COP UVM. Obtenido de https://laureatecomunicacion.com/prensa/covid-19-afecta-estado-emocional-y-economico-dejovenes-en-mexico-cop-uvm/\#.X87LhXBKjIU

Redacción Animal Político . (09 de Agosto de 2020). SEP estima deserción de 10\% en educación básica y $8 \%$ en superior por la COVID. Obtenido de https://www.animalpolitico.com/2020/08/sep-desercion-educacion-covid/

Ruiz, R. (30 de Marzo de 2020 ). Analizan expertos efectos del COVID-19 en la educación pública. Obtenido de https://tec.mx/es/noticias/ciudad-demexico/educacion/analizan-expertos-efectos-del-covid-19-en-la-educacionpublica 